\title{
Trombosis venosa cerebral en la Unidad de Cuidados Intensivos Neurológicos del Instituto Nacional de Neurología y Neurocirugía**
}

Brain venous thrombosis in the unit of neurological intensive care of the National Institute of Neurology and Neurosurgery Trombose venosa cerebral na Unidade de Terapia Intensiva Neurológica do Instituto Nacional de Neurologia e Neurocirurgia

\author{
Uriel García Cabrera, ${ }^{*}$ José Juan Gracia Vera, ${ }^{\ddagger}$ Guadalupe Juárez Díaz, ${ }^{\S}$ Jocelyn Cruz Pérez," Israel Becerra Hernández"
}

\section{RESUMEN}

La trombosis venosa cerebral (TVC) es un estado potencialmente devastador que ocurre en adultos jóvenes, especialmente en mujeres. Es un subtipo menos frecuente de la enfermedad vascular cerebral (EVC) que representa sólo $0.5 \%$ de los pacientes que tienen enfermedad vascular cerebral. La cefalea es el síntoma más común. Requiere un diagnóstico preciso, ya que la fisiopatología y el tratamiento difieren de la enfermedad vascular cerebral arterial. La comprensión de los factores de riesgo es la clave para el pronóstico de la trombosis venosa cerebral.

Objetivo: Determinar la prevalencia y los principales hallazgos clínicos, radiológicos y pronósticos de la trombosis venosa cerebral en la Unidad de Cuidados Intensivos Neurológicos.

Material y métodos: Estudio retrospectivo, longitudinal y analítico de carácter observacional. Se consideraron todos los pacientes que ingresaron a la Unidad de Cuidados Intensivos Neurológicos del Instituto Nacional de Neurología y Neurocirugía (INNN) con el diagnóstico de trombosis venosa cerebral, de enero de 2010 a julio de 2019, confirmados por tomografía computada fase venosa (veno TC) o venorresonancia magnética (veno RM), y que contaran con información de cuadro clínico y estudios de gabinete, así como con evolución, tratamiento y pronóstico al egreso hospitalario.

Resultados: Hubo un total de 14 pacientes con una edad promedio de 33 años de edad, de los cuales $85.72 \%(n=12)$ fueron mujeres y $14.28 \%(n=2)$ fueron hombres. La cefalea se presentó en $50 \%$ de los casos. La principal causa predisponente para trombosis venosa cerebral fue el uso de anticonceptivos orales en seis pacientes $(42.85 \%$ ) y puerperio en tres pacientes $(21.42 \%)$. La demora en el diagnóstico fue en promedio de 48 horas. El método de imagen utilizado para realizar el diagnóstico fue: tomografía computada en fase venosa en $64.28 \%$ de los pacientes y venorresonancia magnética en $37.71 \%$. El seno longitudinal superior fue el más afectado en $50 \%$ de los casos. El promedio de estancia en la Unidad de Cuidados Intensivos (UCl) fue de siete días, donde $100 \%$ de los pacientes recibieron anticoagulación. Desarrollaron hipertensión intracraneana tres $(21.4 \%)$ pacientes a las cuales se les realizó craniectomía descompresiva entre el segundo y el quinto día de estancia. Los días de ventilación mecánica en promedio fueron de siete días, con una estancia hospitalaria en promedio de 20 días. La mortalidad al egreso hospitalario fue de $21.42 \%$.

Conclusiones: La trombosis venosa cerebral es menos frecuente que el accidente cerebrovascular isquémico o la hemorragia intracerebral. El espectro de la clínica es amplio, teniendo como dato pivote la cefalea. La confirmación del diagnóstico se debe realizar con veno TC o veno RM. La intervención terapéutica dentro de la fase aguda está encaminada a la recanalización del seno o senos trombosados y la prevención de complicaciones; la anticoagulación con heparina de bajo peso molecular es el tratamiento de primera línea, el cual ha mostrado impacto en el pronóstico de los pacientes. Debemos tener en cuenta que la trombólisis y la trombectomía son una opción en el tratamiento. En el caso de la craniectomía descompresiva está indicada sólo en caso de infartos venosos malignos. Los resultados después de la trombosis venosa cerebral son generalmente favorables, pero dependen de factores del paciente, como el sexo y los factores de riesgo específicos de la mujer.

Palabras clave: Trombosis venosa cerebral, enfermedad vascular cerebral.

** Ganador del Premio Académico «Dr. Mario Shapiro» 2019. Segundo lugar.

* Unidad Médica de Alta Especialidad No. 1 del Centro Médico Nacional del Bajío, IMSS. León, Guanajuato.

‡ Unidad Médica de Alta Especialidad No. 1 «Ignacio García Téllez». Mérida, Yucatán.

§ Centro Médico ISSEMyM Toluca. Toluca, Estado de México.

\| Instituto Nacional de Neurología y Neurocirugía (INNN) «Manuel Velasco Suárez». Ciudad de México, México.

Recepción: 30/08/2019. Aceptación: 03/12/2019.

Este artículo puede ser consultado en versión completa en www.medigraphic.com/medicinacritica

\section{ABSTRACT}

Cerebral venous thrombosis (TVC) is a potentially devastating state that occurs in young adults, especially women. Less frequent subtype of cerebral vascular disease (EVC), represented only $0.5 \%$ in EVC patients. Headache is the most common symptom common. It requires an accurate diagnosis since the pathophysiology and treatment differ from the arterial EVC. The understanding of the risk factors is the key to the prognosis of the TVC.

Objective: To determine the prevalence, the main clinical, radiological findings and prognosis of TVC in Neurological Intensive Care Unit.

Material and methods: Retrospective, longitudinal and analytical study of an observational nature. All patients admitted to the Neurological Intensive Care Unit of the National Institute of Neurology and Neurosurgery (INNN) with the diagnosis of TVC from January 2010 to July 2019 confirmed by venous phase computed tomography (vein CT) and/or vein were considered MRI (vein MRI), which will have clinical information and cabinet studies, as well as, the evolution, treatment and prognosis at hospital discharge.

Results: Of a total of 14 patients, with an average age of 33 years of age. Of which $85.72 \%(n=12)$ were women and $14.28(n=2)$ were men. Headache occurred $50 \%$ of cases. The main predisposing cause for TVC was the use of oral contraceptives in 6 patients (42.85\%) and puerperium in three patients $(21.42 \%)$. The delay in diagnosis was an average of 48 hours. The imaging method used for diagnosis in $64.28 \%$ of those with computed tomography in venous phase and in $37.71 \%$ with vein magnetic resonance. The upper longitudinal sinus was the most affected in $50 \%$ of cases. The average stay in the Intensive Care Unit (ICU) was seven days, where $100 \%$ of patients received anticoagulation. Three patients $(21.4 \%)$ developed intracranial hypertension who underwent decompressive craniectomy between the second and fifth day of stay. The days of mechanical ventilation on average were seven days. With an average hospital stay of 20 days. Mortality at hospital discharge was $21.42 \%$.

Conclusions: TVC is less frequent than ischemic stroke or intracerebral hemorrhage. The spectrum of the clinic is broad, with pivotal headache. The confirmation of the diagnosis must be performed with CT vein and/or RM vein. The therapeutic intervention within the acute phase is aimed at the recanalization of the thrombosed sinus or sinuses and the prevention of complications; anticoagulation with low molecular weight heparin is the first-line treatment, which has shown an impact on the prognosis of patients. We must keep in mind that thrombolysis and thrombectomy are an option in treatment. In the case of decompressive craniectomy, it is indicated only in cases of malignant venous infarctions. The results after TVC are generally favorable, they also depend on the patient's factors, such as sex and the specific risk factors of women.

Keywords: Cerebral venous thrombosis, cerebral vascular disease.

RESUMO

Trombose venosa cerebral (TVC) é um estado potencialmente devastador que ocorre em adultos jovens, especialmente mulheres. Subtipo menos frequente do acidente vascular cerebral (AVC), representou apenas $0.5 \%$ nos pacientes com AVC. Cefaléia é o sintoma mais comum. Exige um diagnóstico preciso, pois a fisiopatologia e o tratamento diferem da AVC arterial, e a compreensão dos fatores de risco é a chave para o prognóstico da TVC.

Objetivo: Determinar a prevalência, os principais achados clínicos, radiológicos e prognósticos da TVC na unidade de terapia intensiva neurológica.

Material e métodos: Estudo retrospectivo, longitudinal e analítico de natureza observacional. Todos os pacientes admitidos na unidade de terapia intensiva neurológica do Instituto Nacional de Neurologia e Neurocirurgia (INNN) com diagnóstico de TVC de janeiro de 2010 a julho de 2019 confirmados por tomografia computadorizada de fase venosa (Veno-TC) elou veno ressonância magnética (veno-RM), que contaram com informações clínicas e estudos de gabinete, bem como a evolução, tratamento e prognóstico na alta hospitalar.

Resultados: Do total de 14 pacientes, com idade média de 33 anos. Dos quais $85.72 \%(n=12)$ eram mulheres e $14.28(n=2)$ eram homens. $A$ cefaléia ocorreu em $50 \%$ dos casos. A principal causa predisponente 
para TVC foi o uso de contraceptivos orais em 6 pacientes $(42.85 \%)$ e puerpério em 3 pacientes (21.42\%). O atraso no diagnóstico foi em média de 48 horas. 0 método de imagem utilizado para o diagnóstico em $64.28 \%$ daqueles com tomografia computadorizada na fase venosa e em $37.71 \%$ com veno ressonância magnética. O seio sagital superior foi o mais acometido em $50 \%$ dos casos. A permanência média na unidade de terapia intensiva (UTI) foi de 7 dias, onde $100 \%$ dos pacientes receberam anticoagulação. Três pacientes (21.4\%) desenvolveram hipertensão intracraniana submetidos a craniectomia descompressiva entre o segundo e o quinto dia de internação. Os dias de ventilação mecânica foram em média 7 dias. Com internação média de 20 dias. A mortalidade na alta hospitalar foi de $21.42 \%$.

Conclusões: A TVC é menos frequente que o AVC isquêmico ou hemorragia intracerebral. O espectro da clínica é amplo, tendo como sintoma principal cefaléia. A confirmação do diagnóstico deve ser realizada com veno TC e/ou veno RM. A intervenção terapêutica na fase aguda visa à recanalização do seio ou seios trombosados e à prevenção de complicações; a anticoagulação com heparina de baixo peso molecular é o tratamento de primeira linha que demonstrou um impacto no prognóstico dos pacientes. Devemos ter em mente que trombólise e trombectomia são uma opção no tratamento. No caso da craniectomia descompressiva, é indicado apenas em casos de infartos venosos malignos. Os resultados após o TVC são geralmente favoráveis, também dependem de fatores do paciente, como sexo e fatores de risco específicos da mulher.

Palavras-chave: Trombose venosa cerebral, doença vascular cerebral.

\section{INTRODUCCIÓN}

La trombosis venosa cerebral (TVC) es un tipo de accidente cerebrovascular poco frecuente pero potencialmente devastador, caracterizado por la oclusión de la circulación venosa del cerebro. Ésta afecta a adultos jóvenes principalmente, pero es predominante en mujeres. Representa sólo alrededor de $0.5 \%$ de los pacientes con accidente cerebrovascular y tiene una prevalencia de sólo 5 por 1 millón. ${ }^{1}$

En México, Rodríguez Rubio L. y colaboradores reportaron que la edad promedio de presentación es a los 30 años. Las mujeres representaron $83 \%$ de los casos, donde la cefalea y los signos focales fueron las manifestaciones más comunes y el puerperio la condición más frecuentemente asociada con la TVC. ${ }^{2}$

Los anticonceptivos orales se han considerado un factor que contribuye al desarrollo de TVC y su uso se ha informado en 54 a $71 \%$ de los pacientes con TVC. Los factores de riesgo transitorios para la trombosis venosa cerebral incluyen embarazo/puerperio, SNC o infecciones de oído, seno, boca, cara, exposición a medicamentos (esteroides y tratamientos contra el cáncer), traumatismo craneal o procedimientos (por ejemplo, punción lumbar y colocación de catéter yugular). Los desencadenantes crónicos de la trombosis venosa cerebral incluyen trombofilias hereditarias o adquiridas que son causas establecidas de tromboembolismo venoso. ${ }^{3}$

Desde hace tiempo se sabe que los anticonceptivos orales están relacionados con un mayor riesgo de desarrollar tromboembolismo venoso, especialmente en pacientes portadores de defectos tromboembólicos hereditarios comunes como el factor $V$ Leiden, el polimorfismo del factor de protrombina G20210A, la mutación MTHFR (C677T) y el polimorfismo 4G/5G del gen
PAl-1, que representan la mayoría de los eventos tromboembólicos asociados con el uso de anticonceptivos orales; también, el uso de preparaciones que contienen progestinas de tercera generación, trombofilia debido a antitrombina, deficiencia de proteína $\mathrm{C}$ y S, factor homocigoto V (Leiden) y defectos combinados. Puede ocurrir un aumento en los niveles de factores procoagulantes, como los factores VII, X, XII y XIII, asociados con el uso de estrógenos y una reducción en los factores anticoagulantes, incluidas la proteína $S$ y la antitrombina. ${ }^{4,5}$

Con frecuencia no se diagnostica lo suficiente, ya que las manifestaciones clínicas no son específicas e incluyen una amplia gama de síntomas como dolor de cabeza, déficits neurológicos focales, convulsiones y estado mental alterado. La presentación aguda (es decir, menos de 2 días), subaguda (es decir, de 2 días a 1 mes) y crónica (es decir, más de 1 mes) se observa en 30,50 y $20 \%$ de los pacientes, respectivamente. ${ }^{6}$

Se puede clasificar en cuatro síndromes clínicos: 1 ) déficit neurológico; 2) convulsiones que se pueden o no deber a un déficit neurológico focal; 3) Encefalopatía e 4) hipertensión intracraneal. ${ }^{7}$

La neuroimagen desempeña un papel fundamental en el diagnóstico de TVC, lo que influye en el pronóstico y el resultado del paciente. Los hallazgos radiológicos son diversos y dependen de la modalidad de imagen elegida. Esencialmente, estos hallazgos se pueden dividir en signos directos e indirectos, dependiendo de la capacidad para demostrar el trombo o ilustrar los hallazgos asociados, como hemorragia intracraneal o isquemia. $^{8}$

La tomografía computarizada sin contraste suele ser el examen de primera elección en el diagnóstico de los trastornos agudos del SNC. La hiperdensidad del seno dural, la vena cortical (signo del cordón) o las venas profundas es característica de la TVC debido al aumento de la densidad del trombo en comparación con el flujo sanguíneo. ${ }^{9}$

La venorresonancia magnética (veno RM) es útil no sólo para la documentación del trombo, sino también para la evaluación de la recanalización después de la terapia, y en el contexto de trombosis crónica con recanalización parcial. La venorresonancia magnética con gadolinio permite una evaluación directa del relleno luminal similar al de la venografía por tomografía (veno TC), con una sensibilidad y especificidad comparables. La venorresonancia magnética 3D con contraste estático permite una mejor caracterización de la anatomía venosa intracraneal, sin embargo, puede tener limitaciones en la trombosis crónica del seno dural, ya que el coágulo puede aumentar, simulando un seno abierto. Una desventaja significativa de la veno RM en comparación con la veno TC es que los tiempos de adquisición son largos, y pueden producirse artefactos de movimiento en pacientes con bajo nivel de conciencia. ${ }^{10-12}$ 
Tabla 1: Principales manifestaciones clínicas de la trombosis venosa cerebral al ingreso hospitalario $(n=14)$.

\begin{tabular}{ll}
\hline Síntoma & $\mathrm{n}(\%)$ \\
\hline Cefalea & $7(50.00)$ \\
Convulsiones & $3(21.42)$ \\
Disminución en el estado de conciencia & $3(21.42)$ \\
Déficit motor & $1(7.14)$ \\
\hline
\end{tabular}

El tratamiento agudo de la TVC implica la recanalización y la prevención de complicaciones. Deben vigilarse los signos de aumento de presión intracraneal e iniciar su tratamiento con medidas generales (elevación de la cabeza, normocapnia o hipocapnia leve permisiva, tratamiento osmótico o drenaje ventricular en caso de ser necesarios)..$^{1,13}$

La anticoagulación es el tratamiento estándar para prevenir el crecimiento de trombos, facilitar la recanalización y prevenir otros eventos trombóticos siempre y cuando no existan contraindicaciones. La heparina de bajo peso molecular (HBPM) es más segura y eficaz que la heparina no fraccionada, excepto cuando se anticipa la reversión rápida de la anticoagulación debida a una intervención neuroquirúrgica. ${ }^{14-16}$

La terapia endovascular incluye la fibrinólisis sistémica, la trombólisis intravascular local y la trombectomía mecánica venosa, y se considera en el caso de deterioro neurológico progresivo a pesar del tratamiento médico intensivo. La trombólisis está considerada para los pacientes que no tuvieron éxito con la anticoagulación. La trombectomía mecánica es otra alternativa para mejorar la recanalización. El dispositivo más utilizado es el AngioJet, seguido por venoplasty globo sin colocación de stent y el sistema penumbra. La hemicraniectomía descompresiva debe reservarse para pacientes con TVC ante un infarto cerebral hemisférico maligno y deterioro neurológico progresivo, y datos de herniación secundaria a hipertensión intracraneana. ${ }^{17-19}$

Dentro de las complicaciones reportadas están las crisis convulsivas, las cuales pueden ocurrir hasta en $39 \%$ de los casos, de acuerdo con lo reportado en el International Study on Cerebral Vein and Dural Sinus Thrombosis (ISCVT). La hidrocefalia se ha reportado en $15 \%$ de los casos, la cual se presenta principalmente cuando se ven afectados los senos sagital superior y senos trasversales, debido a que se puede afectar el drenaje del líquido cefalorraquídeo (LCR). El tratamiento apropiado incluye medidas para disminuir la producción de LCR, la recanalización del seno trombosado y el manejo de la hipertensión intracraneal. Puede ser necesaria la consulta neuroquirúrgica para la evacuación del LCR con ventriculostomía o derivación ventriculoperitoneal. En el caso de la hipertensión intracraneal, generalmente es causada por obstrucción del flujo venoso y malabsorción del LCR. La hiperten- sión intracraneal se puede observar en 15 a $40 \%$ de los pacientes con TVC. ${ }^{20,21}$

La recurrencia de cualquier evento trombótico después de una TVC es de aproximadamente $6.5 \%$ por año..$^{1,21}$

\section{MATERIAL Y MÉTODOS}

Estudio retrospectivo, longitudinal y analítico de carácter observacional. Se consideraron todos los pacientes que ingresaron a la Unidad de Cuidados Intensivos Neurológicos del Instituto Nacional de Neurología y Neurocirugía (INNN) de enero del 2010 a julio 2019, confirmados por tomografía axial computarizada fase venosa y venorresonancia magnética.

Todos los pacientes contaron con la información clínica relativa a los aspectos demográficos (edad y género), manifestaciones neurológicas al ingreso, tratamiento y evolución durante su estancia en la UCl y tratamiento intrahospitalario.

Los datos demográficos se presentan como frecuencia relativa simple a través de tendencia central. Se empleó el paquete estadístico Excel 2013.

\section{RESULTADOS}

De enero del 2010 a julio del 2019 fueron ingresados a la Unidad de Cuidados Intensivos Neurológicos 14 pacientes con el diagnóstico de TVC, de ellos 12 fueron mujeres $(85.72 \%)$ y 2 hombres $(14.28 \%)$. La principal manifestación clínica fue la cefalea, convulsiones, déficit motor y alteración del estado de conciencia (Tabla 1).

La veno TC de cráneo fue realizada de forma inicial en prácticamente en $100 \%$ de los pacientes y se efec-

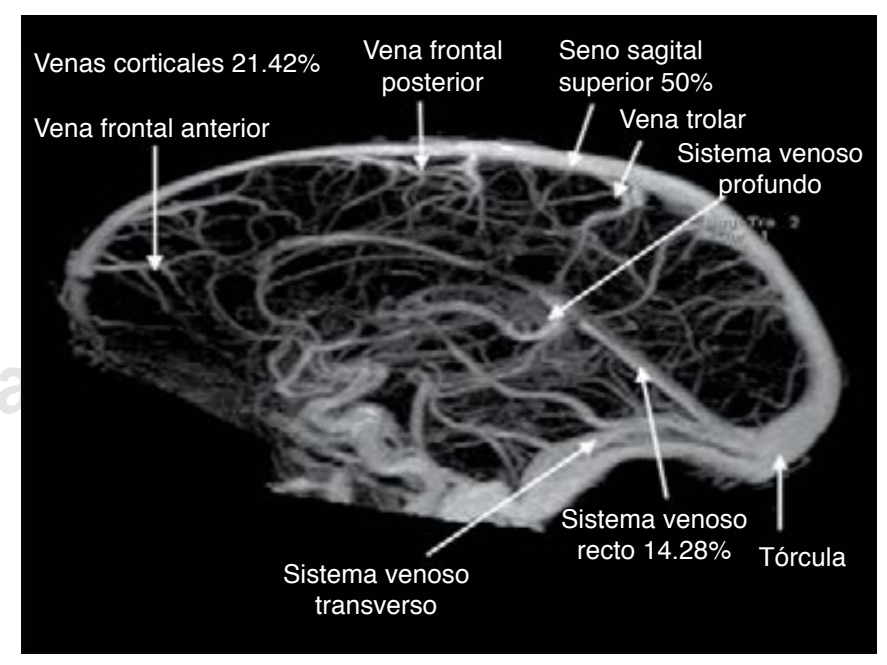

Figura 1: Trombosis venosa cerebral. Senos afectados más comúnmente. Tomada de: Bushnell C, Saposnik G. Evaluation and management of cerebral venous thrombosis. Continuum (Minneap Minn). 2014;20(2 Cerebrovascular Disease):335-351. 
tuó el diagnóstico en nueve pacientes (64.24\%) y venorresonancia magnética en cinco pacientes (35.71\%). Sólo en dos pacientes se realizó angiografía cerebral, aunque en ellos, ya había orientación diagnóstica de la veno TC y también a través de la veno RM (Figura 1). El principal seno afectado fue el seno longitudinal superior en siete pacientes $(50 \%)$, seguido de las venas corticales en tres pacientes (21.42\%), el seno recto en dos pacientes (14.28\%) y el seno transverso en dos pacientes (14.28\%) (Figura 2). La demora en el diagnóstico fue en promedio de 48 horas.

La principal causa predisponente para TVC fue el uso de anticonceptivos orales en seis pacientes (42.85\%) y puerperio en tres pacientes (21.42\%) (Tabla 2). En el caso de las TVC asociadas con puerperio, éstas se presentaron durante la fase tardía del mismo.

Cien por ciento de los pacientes recibieron tratamiento de enoxaparina dentro de las primeras 48 horas de su ingreso y continuaron con anticoagulantes orales en su egreso hospitalario. Otros fármacos utilizados en la fase aguda fueron: los anticonvulsivantes en tres pacientes $(21.42 \%)$, norepinefrina en tres pacientes (21.42\%), midazolam en seis pacientes $(42.48 \%)$, propofol en tres pacientes $(21.42 \%)$ y esteroides en dos pacientes (14.28\%).

El promedio de estancia en la Unidad de Cuidados Intensivos fue de siete días, donde $100 \%$ de los pacientes recibieron anticoagulación. Tres pacientes (21.4\%) desarrollaron hipertensión intracraneana, a los cuales se les realizó craniectomía descompresiva entre el segundo y el quinto día de estancia. Los días de ventilación mecánica en promedio fueron de siete días, con una estancia hospitalaria en promedio de 20 días.

Además, se presentó una mortalidad al egreso hospitalario de tres pacientes $(21.42 \%)$, las cuales ocurrieron en una paciente de 19, 33 y 35 años de edad, respectivamente, dos asociadas con el consumo de anticonceptivos orales y la de un paciente sin causa determinada. Dos pacientes ingresaron por deterioro neurológico y una por crisis convulsiva; dos con afectación del seno longitudinal superior y una con afectación del seno transverso y seno longitudinal superior, ambas confirmadas por venorresonancia magnética y desarrollaron datos de hipertensión intracraneana. Ésta fue documentada por cuadro clínico y estudio tomográfico, lo que evidenció desviación de línea media $\geq 10 \mathrm{~mm}$, por lo que las pacientes fueron sometidas a craniectomía descompresiva.

Cien por ciento de los pacientes al ingreso tuvieron una escala de Rankin modificada de 0 . Durante el seguimiento, nueve pacientes (64.28\%) presentaron evolución favorable; se encontraron asintomáticas en su última visita de seguimiento dos pacientes (14.28\%); éstas tenían secuelas menores, y reportaron Rankin de 1 y 2 en su consulta de seguimiento. Sólo tres pacientes
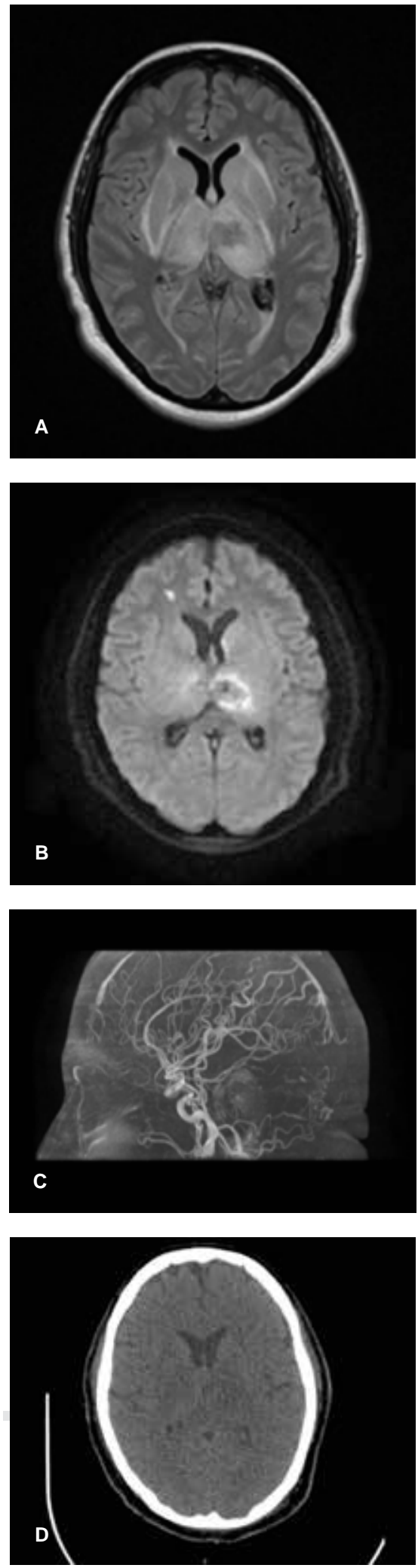

Figura 2: A) Angiorresonancia T2 Flair 2. Signo del Delta vacío. B) Angiorresonancia en difusión. Edema y sangrado talámico izquierdo. C) Angiorresonancia 3D TOF. Retardo de llenado del seno sagital inferior y seno recto. D) TAC de cráneo simple. Recanalización de la tórcula. 
Tabla 2: Condición predisponente para trombosis venosa cerebral $(n=14)$.

\begin{tabular}{ll}
\hline Condición predisponente & $\mathrm{n}(\%)$ \\
\hline Anticonceptivos orales & $6(42.85)$ \\
No determinado & $4(28.57)$ \\
Puerperio & $3(21.42)$ \\
Hipotiroidismo & $1(7.14)$ \\
\hline
\end{tabular}

(21.42\%) presentaron Rankin de 6 durante su estancia en la UCl.

\section{DISCUSIÓN}

La TVC es un tipo de enfermedad vascular cerebral (EVC) pero potencialmente devastador que ocurre en adultos jóvenes, especialmente en mujeres. Representa sólo $0.5 \%$ de los pacientes con EVC. ${ }^{1}$ En México ha sido reportada con una frecuencia menor de $10 \%$ en poblaciones donde se incluyeron pacientes ingresados a Servicio de Medicina Interna y Unidad de Cuidados Intensivos. ${ }^{2}$ La prevalencia observada en nuestra unidad fue de $0.75 \%$ del total de ingresos de la UCl.

Dentro de las condiciones predisponentes para TVC, de acuerdo con lo reportado en la literatura, una condición es la de anticonceptivos orales en $54-71 \%$ de los casos aproximadamente, lo cual no difiere de lo reportado en el presente estudio (Tabla 1), seguida del embarazo y el puerperio. 1,3 En México, en 2009 se reportó que el puerperio era la principal causa predisponente para TVC. ${ }^{2}$

Para el diagnóstico es importante mantener una sospecha clínica con especial énfasis en pacientes jóvenes que cuenten con factores de riesgo como los previamente mencionados. De acuerdo con lo reportado por Cheryl B. y colaboradores en el 2014, la cefalea es el síntoma predominante, pues representa hasta $90 \%$ de los casos, ${ }^{1}$ lo que no difiere con lo reportado en un estudio en México en el $2009^{2}$ y en el presente.

En la TVC se pueden observar datos indirectos como infartos o sangrados que no representan territorios arteriales en la TC, por lo que el diagnóstico se debe realizar mediante veno TC o veno RM, de acuerdo con lo recomendado por la European Federation of Neurological Societies (EFNS) y la American Heart Association/ American Stroke Association. ${ }^{22,23}$ En nuestra población los estudios de gabinete se usaron para la confirmación del diagnóstico.

La terapia con anticoagulantes es controversial, sin embargo, es la terapéutica que ha mostrado mejorar el pronóstico, inclusive en presencia de hematomas francos o infartos venosos hemorrágicos de acuerdo a lo recomendado por la American Heart Association Guidelines, European Federation of Neurological Societies Guidelines y la American College of Chest Physicians
Guidelines que le dan un nivel de recomendación clase Ila, nivel B y grado IIC respectivamente. ${ }^{20,23,24}$ Por lo que en el presente estudio se inició enoxaparina en $100 \%$ de los pacientes, al ser confirmado el diagnóstico, el cual no fue más allá de 48 horas posteriores a su ingreso.

La terapia endovascular se considera en aquellos pacientes con deterioro neurológico a pesar del tratamiento establecido, incluida la anticoagulación, sin embargo, hay muy poca evidencia actual. ${ }^{1,22,23}$ Dentro de los pacientes incluidos en este estudio, ninguno recibió terapia endovascular.

Con lo que respecta a la craniectomía descompresiva debe reservarse para pacientes jóvenes con TVC que desarrollan un accidente cerebrovascular hemisférico maligno con deterioro neurológico progresivo, a pesar del tratamiento médico intensivo. En otra revisión que incluyó a 13 pacientes con TVC grave que se sometieron a hemicraniectomía descompresiva, 11 $(84.6 \%)$ lograron un resultado favorable (puntuación de la escala de Rankin modificada de tres o menos). ${ }^{1}$ En nuestra población de estudio, se les realizó a tres pacientes $(21.42 \%)$, las cuales, mediante estudio tomográfico, evidenciaron la presencia de desplazamiento de la línea media $\geq 10 \mathrm{~mm}$. Las tres pacientes fallecieron durante su estancia en la Unidad de Cuidados Intensivos.

El pronóstico de la TVC es significativamente menos deletéreo comparado con otros tipos de EVC y conlleva menos mortalidad ( $\leq 15-25 \%$ ) según los reportes, ${ }^{1}$ por lo que, de acuerdo con los resultados encontrados en el desarrollo de este estudio, en nuestra unidad estamos dentro del rango de mortalidad documentado a nivel internacional.

Un episodio de recurrencia de TVC, de acuerdo con lo documentado, puede sucederle a $6.5 \%$ de los pacientes dentro del primer año. ${ }^{2,20,21}$ De acuerdo con nuestro registro fue de $0 \%$ con seguimiento a nueve meses.

\section{CONCLUSIONES}

La TVC es menos frecuente que el accidente cerebrovascular isquémico o la hemorragia intracerebral. El espectro de la clínica es amplio, teniendo como dato pivote la cefalea. La confirmación del diagnóstico se debe realizar con veno TC y/o veno RM. La intervención terapéutica dentro de la fase aguda está encaminada a la recanalización del seno o senos trombosados y la prevención de complicaciones; la anticoagulación con heparina de bajo peso molecular es el tratamiento de primera línea, que ha mostrado impacto en el pronóstico de los pacientes. Debemos tener en cuenta que la trombólisis y la trombectomía son una opción en el tratamiento. En el caso de la craniectomía descompresiva está indicada sólo en caso de infartos venosos malig- 
nos. Los resultados después de la TVC son generalmente favorables, y también dependen de factores del paciente, como el sexo y los factores de riesgo específicos de la mujer.

\section{BIBLIOGRAFÍA}

1. Bushnell C, Saposnik G. Evaluation and management of cerebral venous thrombosis. Continuum (Minneap Minn). 2014;20(2 Cerebrovascular Disease):335-351.

2. Rodríguez-Rubio LR, Medina-Córdova LL, Andrade-Ramos MA, González-Padilla C, Bañuelos-Becerra LJ, Chiquete E, et al. Trombosis venosa cerebral en el Hospital Civil de Guadalajara "Fray Antonio Alcalde". Rev Mex Neuroci. 2009;10(3):177-183.

3. Özdemir HH, Varol S, Akıl E, Acar A, Demir CF. Evaluation of cerebral venous thrombosis secondary to oral contraceptive use in adolescents. Neurol Sci. 2015;36(1):149-153.

4. Bousser MG, Crassard I. Cerebral venous thrombosis, pregnancy and oral contraceptives. Thromb Res. 2012;130 Suppl 1:S19-S22.

5. Radaković B, Goldstajn MS. MTHFR C 677T mutation and 4G/5G PAI-1 polymorphism in patient with polycystic ovarian syndrome. Coll Antropol. 2007;31(3):919-921.

6. Dmytriw AA, Song JSA, Yu E, Poon CS. Cerebral venous thrombosis: state of the art diagnosis and management. Neuroradiology. 2018;60(7):669-685.

7. Bousser MG, Ferro JM. Cerebral venous thrombosis: an update. Lancet Neurol. 2007;6(2):162-170.

8. Chiewvit P, Piyapittayanan S, Poungvarin N. Cerebral venous thrombosis: diagnosis dilemma. Neurol Int. 2011;3(3):e13.

9. Walecki J, Mruk B, Nawrocka-Laskus E, Piliszek A, Przelaskowski A, Sklinda K. Neuroimaging of cerebral venous thrombosis (CVT) - old dilemma and the new diagnostic methods. Pol J Radiol. 2015;80:368-373.

10. Roland T, Jacobs J, Rappaport A, Vanheste R, Wilms G, Demaerel $P$. Unenhanced brain CT is useful to decide on further imaging in suspected venous sinus thrombosis. Clin Radiol. 2010;65(1):34-39.

11. Meckel S, Reisinger C, Bremerich J, Damm D, Wolbers M, Engelter S, et al. Cerebral venous thrombosis: diagnostic accuracy of combined, dynamic and static, contrast-enhanced 4D MR venography. AJNR Am J Neuroradiol. 2010;31(3):527535.

12. Hedderich DM, Ferro JM, Kunz WG. Diagnostic imaging in the management of patients with possible cerebral venous thrombosis: a cost-effectiveness analysis. Neuroradiology. 2019;61(10):1155-1163.

13. Izura-Gómez $M$, Misis Del Campo $M$, Puyalto de Pablo $P$, Castaño-Duque $C$. Mechanical thrombectomy: an alternative for treating cerebral venous sinus thrombosis. Emergencias. 2018;30(2):123-125.

14. Coutinho JM. Cerebral venous thrombosis. J Thromb Haemost. 2015;13 Suppl 1:S238-S44.
15. Verhagen MJ, van Es AC, Lycklama À Nijeholt GJ, Jellema $\mathrm{K}$, Coutinho J, van den Wijngaard IR. Successful mechanical thrombectomy in a comatose patient with cerebral venous sinus thrombosis: a case report. Interv Neuroradiol. 2017;23(4):437440.

16. Pizzi MA, Alejos DA, Siegel JL, Kim BY, Miller DA, Freeman WD. Cerebral venous thrombosis associated with intracranial hemorrhage and timing of anticoagulation after hemicraniectomy. J Stroke Cerebrovasc Dis. 2016;25(9):2312-2316.

17. Omoto K, Nakagawa I, Park HS, Wada T, Motoyama Y, Kichikawa $\mathrm{K}$, et al. Successful emergent endovascular mechanical thrombectomy for pediatric and young adult cerebral venous sinus thrombosis in coma. World Neurosurg. 2019;122:203-208.

18. Cabral de Andrade G, Lesczynsky A, Clímaco VM, Pereira ER, Marcelino PO, Franco A, et al. Cerebral venous sinuses thrombosis in both transverse sinus and torcula: Multistep endovascular treatment and stenting. Interv Neuroradiol. 2017;23(1):84-89.

19. Kumar S, Rajshekher G, Reddy CR, Venkateswarlu J, Prabhakar S. Intrasinus thrombolysis in cerebral venous sinus thrombosis: single-center experience in 19 patients. Neurol India. 2010;58(2):225-229.

20. Ferro JM, Canhão P, Bousser MG, Stam J, Barinagarrementeria F; ISCVT Investigators. Early seizures in cerebral vein and dural sinus thrombosis: risk factors and role of antiepileptics. Stroke. 2008;39(4):1152-1158.

21. Nasr DM, Brinjikji W, Cloft HJ, Saposnik G, Rabinstein AA. Mortality in cerebral venous thrombosis: results from the national inpatient sample database. Cerebrovasc Dis. 2013;35(1):40-44.

22. Saposnik G, Barinagarrementeria F, Brown RD Jr, Bushnell CD, Cucchiara B, Cushman M, et al. Diagnosis and management of cerebral venous thrombosis: a statement for healthcare professionals from the American Heart Association/American Stroke Association. Stroke. 2011;42(4):1158-1192.

23. Einhäupl K, Stam J, Bousser MG, De Bruijn SF, Ferro JM, Martinelli I, et al. EFNS guideline on the treatment of cerebral venous and sinus thrombosis in adult patients. Eur $J$ Neurol. 2010;17(10):1229-1235.

24. Coutinho JM, Ferro JM, Canhão P, Barinagarrementeria F, Bousser MG, Stam J; ISCVT Investigators. Unfractionated or low-molecular weight heparin for the treatment of cerebral venous thrombosis. Stroke. 2010;41(11):2575-2580.

Relación de conflicto de intereses: sin conflicto de intereses.

Correspondencia:

Uriel García Cabrera

Francisco I Madero Núm. 30 B,

Col. Apeo, 61274, Maravatío, Michoacán.

Teléfono: 4432276694

E-mail: uriel_mich@hotmail.com 